April 24, 2012

Bruce Watson, CHP

U.S. Nuclear Regulatory Commission

Two White Flint North

11545 Rockville Pike

Mail Stop: T-8F37

Rockville, MD 20852-2738

\title{
SUBJECT: TECHNICAL BASES AND GUIDANCE FOR THE USE OF COMPOSITE SOIL SAMPLING FOR DEMONSTRATING COMPLIANCE WITH RADIOLOGICAL RELEASE CRITERIA (RFTA NO. 10-008) DCN: 2023-TR-01-0
}

Dear Mr. Watson:

At the request of the U.S. Nuclear Regulatory Commission (NRC), the Oak Ridge Institute for Science and Education (ORISE) has performed literature reviews and proof-of-concept evaluations to develop the enclosed technical guidance. This guidance provides the methodologies and the technical bases for incorporating composite sampling strategies into decommissioning and final status radiological survey plans. To the extent practicable and where applicable, this guidance follows the Multi-Agency Radiation Survey and Site Investigation Manual (MARSSIM) final status survey (FSS) processes for demonstrating compliance with radiological release criteria.

Comments provided on the revised draft have been incorporated into this final report. My contact information is provided below if we may provide further assistance.

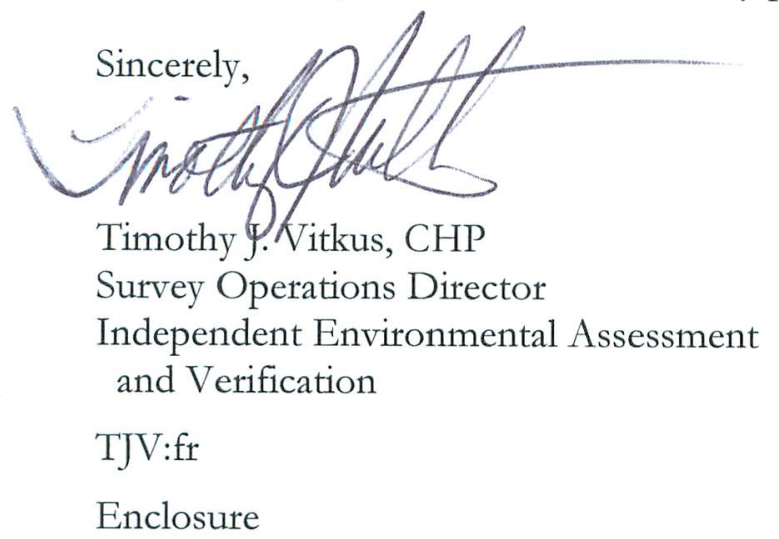

\begin{tabular}{|c|c|}
\hline D. Schmidt, NRC/FSME/DWMEP T-8F5 & E. Bailey, ORISE \\
\hline S. Nesmith, NRC/NMSS/TWFN 8A23 & D. King, ORISE \\
\hline T. Carter, NRC/FSME/DWMEP T-8F5 & P. Weaver, ORISE \\
\hline S. Roberts, ORISE & W. Adams, ORISE \\
\hline
\end{tabular}

File/2023 


\section{TECHNICAL BASES AND GUIDANCE FOR THE USE OF COMPOSITE SOIL SAMPLING FOR DEMONSTRATING COMPLIANCE WITH RADIOLOGICAL RELEASE CRITERIA}

\section{Timothy J. Vitkus}

Prepared for the

U.S. Nuclear Regulatory Committee

OR I S E

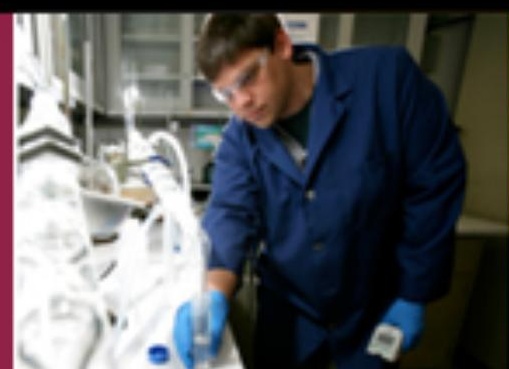


The Oak Ridge Institute for Science and Education (ORISE) is a U.S. Department of Energy institute focusing on scientific initiatives to research health risks from occupational hazards, assess environmental cleanup, respond to radiation medical emergencies, support national security and emergency preparedness, and educate the next generation of scientists. ORISE is managed by Oak Ridge Associated Universities (ORAU).

\section{NOTICES}

The opinions expressed herein do not necessarily reflect the opinions of the sponsoring institutions of Oak Ridge Associated Universities.

This report was prepared as an account of work sponsored by the United States Government. Neither the United States Government nor the U.S. Department of Energy, nor any of their employees, makes any warranty, expressed or implied, or assumes any legal liability or responsibility for the accuracy, completeness, or usefulness of any information, apparatus, product, or process disclosed, or represents that its use would not infringe on privately owned rights. Reference herein to any specific commercial product, process, or service by trade name, mark, manufacturer, or otherwise, does not necessarily constitute or imply its endorsement or recommendation, or favor by the U.S. Government or any agency thereof. The views and opinions of authors expressed herein do not necessarily state or reflect those of the U.S. Government or any agency thereof. 


\title{
TECHNICAL BASES AND GUIDANCE FOR THE USE OF COMPOSITE SOIL SAMPLING FOR DEMONSTRATING COMPLIANCE WITH RADIOLOGICAL RELEASE CRITERIA
}

\author{
Prepared by \\ T.J. Vitkus

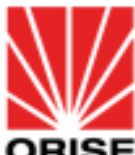 \\ Independent Environmental Assessment and Verification Program \\ Oak Ridge Institute for Science and Education \\ Oak Ridge, Tennessee 37831-0017 \\ Prepared for the \\ U.S. Nuclear Regulatory Commission
}

FINAL REPORT

APRIL 2012

Prepared by the Oak Ridge Institute for Science and Education, under interagency agreement (NRC FIN No. F1008) between the U.S. Nuclear Regulatory Commission and the U.S. Department of Energy. The Oak Ridge Institute for Science and Education performs complementary work under contract number DE-AC05-06OR23100 with the U.S. Department of Energy. 
THIS PAGE LEFT INTENTIONALLY BLANK 


\section{TECHNICAL BASES AND GUIDANCE FOR THE USE OF COMPOSITE SOIL SAMPLING FOR DEMONSTRATING COMPLIANCE WITH RADIOLOGICAL RELEASE CRITERIA}

Prepared by:

T. J. Vitkus IEAX Associate Director, Director Survey Operations

Independent Environmental Assessment and Verification

Reviewed by:

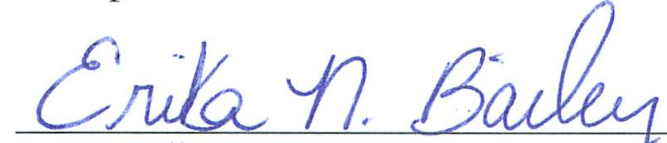

E.N. Bailey, Survey Projects Group Xanager,

Independent Environmental Assessment and Verification

Approved for release by:
Date:

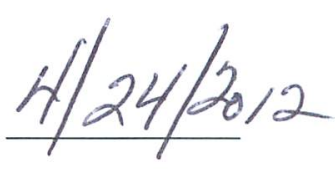

Date: $4 / 24 / 2012$

Date: $4 / 24 / 2012$
E.N. Bailey, Survey Projects Grołtp Manager,

Independent Environmental Assessment and Verification 
THIS PAGE LEFT INTENTIONALLY BLANK 


\section{CONTENTS}

FIGURES ... V

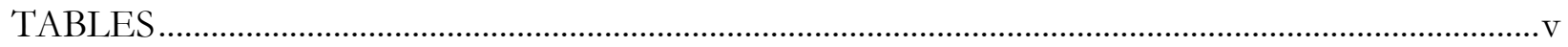

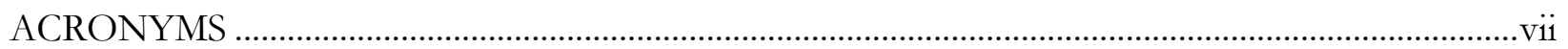

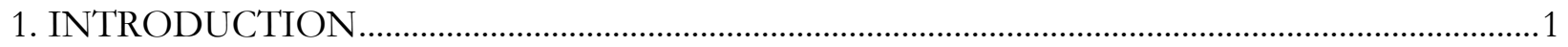

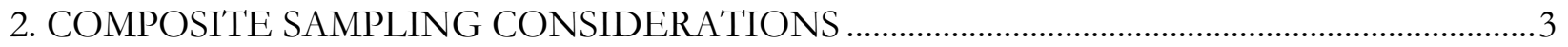

3. COMPOSITE SAMPLE PLAN DESIGN INTEGRATION _............................................................

3.1. USES, CONSIDERATIONS, AND LIMITATIONS FOR COMPOSITE SAMPLING ..........................4

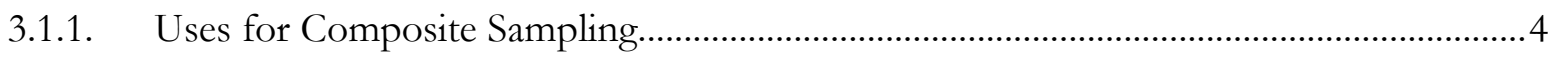

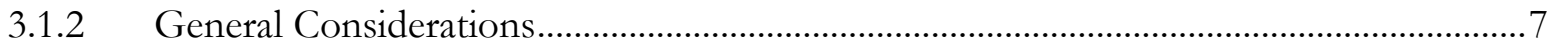

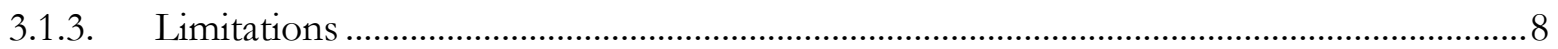

3.2. Composite Sampling Plan And Data Quality AsSESSMENT REVIEW ITEMS ..............9

4. COMPOSITE SAMPLING DESIGNS: SURVEY PLANNING

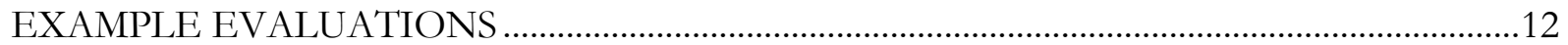

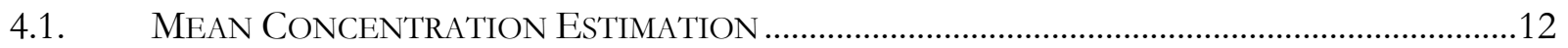

4.2. IdENTIFICATION OF A ROC PRESENCE/AbSENCE TRAIT ….................................................14

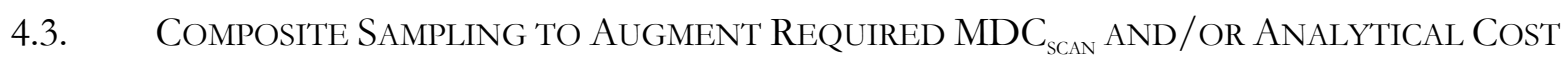

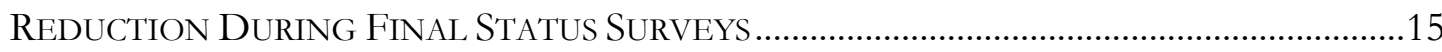

4.4. The Contaminant DCGL $L_{\mathbb{W}}$, MeAn, VAriability, And/Or Decision ERror

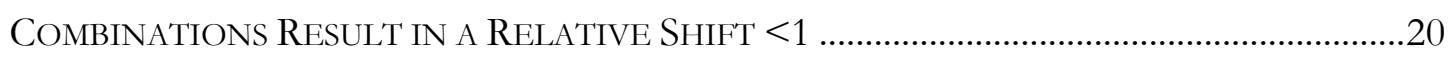

4.5. Classifying Survey Areas As CONTAining Hot SPOTS ..............................................20

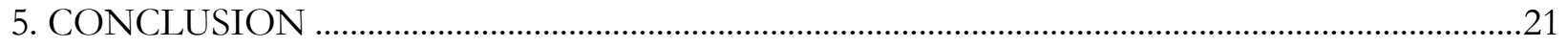

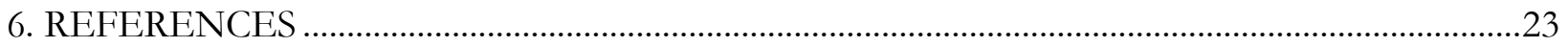


THIS PAGE LEFT INTENTIONALLY BLANK 


\section{FIGURES}

Fig. 4.1. Pu-238 Sample Number Comparison............................................................................................18

\section{TABLES}

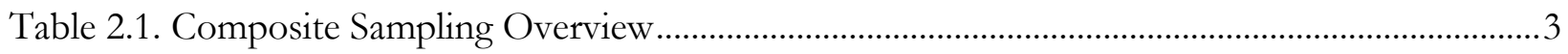

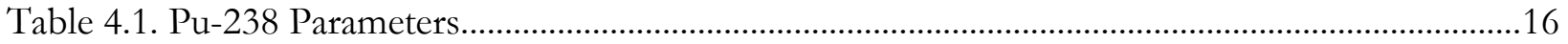

Table 4.2. Composite Result Investigation Level Evaluation................................................................19 
THIS PAGE LEFT INTENTIONALLY BLANK 


\section{ACRONYMS}

DCGL $_{\text {EMC }}$ elevated measurement comparison derived concentration guideline level

DCGL $_{W} \quad$ average derived concentration guideline level

DQO data quality objective

EPA U.S. Environmental Protection Agency

FSS final status survey

HTD hard-to-detect

IL investigation level

LBGR lower bound of the gray region

MARSSIM Multi-Agency Radiation Survey and Site Investigation Manual

MDC minimum detectable concentration

$\mathrm{MDC}_{\mathrm{SCAN}} \quad$ scan minimum detectable concentration

MIL modified investigation level

NRC U.S. Nuclear Regulatory Commission

NUREG Nuclear Regulatory (guidance documents published by the NRC)

ORISE Oak Ridge Institute for Science and Education

$\mathrm{pCi} / \mathrm{g} \quad$ picocuries per gram

ROC radionuclides of concern 
THIS PAGE LEFT INTENTIONALLY BLANK 


\section{TECHNICAL BASES AND GUIDANCE FOR THE USE OF COMPOSITE SOIL SAMPLING FOR DEMONSTRATING COMPLIANCE WITH RADIOLOGICAL RELEASE CRITERIA}

\section{INTRODUCTION}

The Multi-Agency Radiation Survey and Site Investigation Manual (MARSSIM) integrates a flexible, statistically-based final status survey (FSS) sample size- that is, the sample size required to demonstrate compliance with the average derived concentration guideline level (DCGL $\mathrm{W}_{\mathrm{W}}$ ) — together with surface scanning (NRC 2000). The surface scanning specifications for Class 1 survey units include a required scan minimum detectable concentration $\left(\mathrm{MDC}_{\mathrm{SCAN}}\right)$ that is a function of the sample spacing and the respective elevated measurement comparison derived concentration guideline level (DCGL EMC $_{\text {) }}$ that provides assurance that hot spots of concern are identified via either sampling or scanning. For land area survey units (soil), this approach relies upon the radionuclides of concern (ROCs) being gamma emitters and for the case of non-gamma emitting ROCs-i.e., the hard-to-detect ROCs (HTD)—reliance upon a surrogate relationship. However, this specific MARSSIM integrated guidance of coupling sample spacing with a required $\mathrm{MDC}_{\mathrm{SCAN}}$ cannot specifically be followed when the scenario involves HTDs as the only ROCs or when a surrogate relationship cannot be established. In the case of HTDs or certain detectable contaminants in soil where the required $\mathrm{MDC}_{\mathrm{SCAN}}$ is much lower than the actual $\mathrm{MDC}_{\mathrm{SCAN}}$ or in circumstances that result in a relative shift less than 1 , an inordinate and economically burdensome number of samples may be required.

Licensees responsible for sites with HTDs, or situations where the relative shift drops below a value of 1, or instances when the actual $\mathrm{MDC}_{\mathrm{SCAN}}$ for hot spot detection is much greater than the required $\mathrm{MDC}_{\mathrm{SCAN}}$, must rely upon the MARSSIM flexibility in demonstrating compliance with Title 10, Code of Federal Regulations, Part 20, Subpart E license termination as detailed in NUREG-1757 Volume 2, Section 2 (U.S. Nuclear Regulatory Commission [NRC] 2006). Such instances have resulted in some sites proposing to incorporate composite sampling into the FSS sampling strategy to reduce the total number of samples that are analyzed and hence the analytical cost. Neither MARSSIM nor the companion regulatory guidance documents, NUREG-1505 and NUREG-1757, provide specific guidance methodologies for applying composite sampling to an FSS (NRC 2006). Rather, MARSSIM recommends that if an inordinate number of samples are required, then the data 
quality objectives (DQOs) must be revisited. Chapter 14 of NUREG-1505 briefly introduces the concept of composite sampling as a means to reduce the total number of samples requiring analysis (NRC 1998).

This guidance provides the additional information on methodologies and the technical bases that licensees should consider for incorporating composite sampling strategies into FSS plans. In addition, this guidance also includes appropriate uses of composite sampling for generating the data for other decommissioning site investigations such as characterization or other preliminary site investigations.

The composite sampling applications are not limited to just FSS applications but also may be applicable during the site investigation process where composite samples are collected to estimate the mean concentrations, determine ROC distributions, or identify the absence/presence of another specific trait within a study area. The focus of this guidance, therefore, is to link the industryaccepted resources into a concise approach for licensee consideration in designing decommissioning and final status surveys, assessing resultant data, and for the regulatory authority review of proposed radiological surveys that include composite sampling.

Lastly, the FSS-related portions of this guidance follow MARSSIM processes for demonstrating compliance with radiological release criteria and the average allowable residual contamination levels. However, additional evaluations will be necessary to guide the use of composite sampling within the MARSSIM framework for the specific case of ensuring that hot spots of concern are addressed when HTDs are a primary consideration. There are both Federal agency and academic resources available for supplementing the MARSSIM radiological survey processes with composite sampling and the challenges presented by the HTDs. These resources, coupled with the experience and methods gained over the years at chemically contaminated sites, provide the bases for the general approaches that have been included within later sections of this guidance that are applicable to identifying HTD hot spots. Specifically, a literature review of academic statistical composite sampling publications was conducted. Relevant information from these publications was combined with an evaluation of the guidance and recommendations detailed in the following references: the U.S. Environmental Protection Agency's (EPA) Quality System General Guidance-several of these publications are referenced within MARSSIM — and the EPA's Observational Economy Series 
documents (EPA 2002 and 1995). The pedigree of the publications reviewed was also assessed and a clear path was evident in that the academic publications were sources for the Federal guidance documents, leading to other industry publications expanding on the guidance, and finally to incorporation of the expanded information into many of the available software applications used for planning and assessing environmental data (Patil 2002a, Correl 2001, Carson 2001).

\section{COMPOSITE SAMPLING CONSIDERATIONS}

There are scenarios where it could be advantageous for licensees to apply a composite sampling approach for the MARSSIM-based FSS data quality objectives and the associated data life cycle. These scenarios normally will involve a specific set of conditions where the approach could be beneficial. Generally, such conditions would involve situations where the analytical costs are high, required $\mathrm{MDC}_{\mathrm{SCAN}}$ in Class 1 survey units are difficult to achieve, and/or the presence of HTDs both increased analytical costs and reduced detection capability. Therefore, successful implementation of composite sampling requires a well thought-out plan and is normally only beneficial when the conditions above exist. Table 2.1 summarizes when composite sampling is advantageous. The advantages numbered 1,2, and 4 would be applicable for an FSS, and all of the advantages listed are applicable to other survey types, such as site characterization. The disadvantages listed must also be considered and addressed in the planning and data life cycle. Table 2.1 also summarizes the uses and considerations that the site would include in their survey plan in order to provide the regulatory assurance that composite sampling would not interfere with the decision as to whether or not the radiological release criteria are satisfied.

\begin{tabular}{|l|l|}
\hline \multicolumn{2}{|c|}{ Table 2.1. Composite Sampling Overview } \\
\hline \multicolumn{1}{|c|}{ Advantages } & \multicolumn{1}{c|}{ Disadvantages } \\
\hline 1. Reduces analytical costs. & $\begin{array}{l}\text { 1. Should not be used for establishing surrogate } \\
\text { ratios. }\end{array}$ \\
\hline $\begin{array}{l}\text { 2. Provides a better estimate of mean concentration } \\
\text { in the study area. }\end{array}$ & $\begin{array}{l}\text { 2. Information is lost on the individual sample } \\
\text { increments that make up a composite. This loss of } \\
\text { information is a concern when testing to } \\
\text { determine if a ROC exceeds a threshold, e.g., a } \\
\text { DCGLEMC Over a specific area because of possible } \\
\text { dilution to one or more increments with elevated } \\
\text { activity concentrations by the other composite } \\
\text { increments. }\end{array}$ \\
\hline
\end{tabular}




\section{Table 2.1. Composite Sampling Overview}

\section{Advantages}

Disadvantages

3. Cannot be used when action levels (DCGLws) are near analytical detection limits or the natural background concentration levels.

3. Identifying units that have the highest contaminant levels.

4. With an appropriately adjusted contaminant benchmark/investigation level, composite sampling can increase the ability to detect hot spots by increasing the number of locations sampled.
4. For non-homogenous contaminant distributions, temporal or spatial variability information is lost.

5. Cannot be used when integrity of individual sample values change, such as loss of volatile contaminants, due to the physical compositing mechanism.

\section{Uses and Considerations for Applying Composite Sampling}

1. Useful when the size of the pattern or feature of interest, such as hot spots, is smaller than the spacing between the statistically required random sampling locations.

2. User must account for potential introduction of large additional errors due to heterogeneous nature of the contaminant in the matrix, or the matrix itself.

3. Aliquots used to form the composite must be of equivalent weight/volume and the individual aliquots and the composite itself must be well homogenized.

4. Must account for the dilution factor when evaluating the result against a threshold, most commonly a hotspot or legal action threshold. Necessitates a modified investigation level (MIL).

5. In most cases, the user must maintain the ability for re-testing of individual samples (increments) making up the composite to retrieve potentially lost information.

\section{COMPOSITE SAMPLE PLAN DESIGN INTEGRATION}

This examination first reviews the conditions under which a composite sampling approach is appropriate and would be considered advantageous, and concurrently reviews those conditions which may impact decisions made during the data quality assessment phase.

\subsection{USES, CONSIDERATIONS, AND Limitations FOR COMPOSITE SAMPLING}

This guidance considers the application of composite sampling to address the following decommissioning survey conditions and general limitations.

\subsubsection{Uses for Composite Sampling}

1. Using composite sampling to estimate the mean concentration of an ROC would be 
applicable to various decommissioning survey types including scoping, characterization, remedial action support, and final status. However, the data user must recognize that although an equivalent or better estimate of the mean with fewer analyzed samples is an outcome of compositing versus individual sample analyses, there is a loss of crucial information as to the extent of the overall variability of the ROCs within the study area. This is due to inherent "smoothing" of the uncertainty that results from compositing. A simple example may be used to illustrate this factor.

- 10 locations_-numbered 1 through 10 — are selected for random sampling from the study area.

- The analytically-determined concentrations at these 10 locations are $1,2,3 \ldots 10 \mathrm{pCi} / \mathrm{g}$ (picocuries per gram), respectively.

- The calculated study area mean and standard deviation (sigma) would be calculated to be $5.5 \pm 2.9$.

- Next, assume 2 composites are formed and analyzed. Composite 1 from the even numbered locations and Composite 2 from the odd numbered locations. The composite concentrations would be 5 and 6 , respectively. The study area mean and sigma would be estimated to be $5.5 \pm 0.5$.

This example illustrates that the mean is as accurately estimated with 2 composites; however, the actual study area variability is significantly underestimated. This factor will be an important consideration should the data be used in the construction of an upper confidence level for the study area (i.e., survey unit/investigation area) or the number of samples required to ensure adequate power for hypothesis tests. Therefore, the user will need to evaluate the balance between the number of samples (n) required for statistical considerations and the number of increments (defined as $k$ ).

2. Compositing is useful for estimating the proportion of a population exhibiting a trait, such as the presence or absence of a specific ROC.

3. It can be used to classify survey areas as containing hot spots or identify survey area ROC 
concentration level spatial distributions, such as those parts of the site with the highest concentration levels.

4. Composite sampling can be used when the contaminant DCGL $\mathrm{W}_{\mathrm{W}}$, mean, variability, and/or decision error combinations result in a relative shift $<1$ or otherwise requires an inordinate number of samples. The site may elect to use composite sampling to reduce the number of samples requiring analysis, yet still meet the sample number to adequately estimate the survey unit mean/median for the selected statistical test.

5. Composite sampling may be used as a method to decrease sample spacing. This may occur when the ROC may be a low abundance or low-energy gamma emitter with a high MDC $_{\text {SCAN }}$ relative to the required $\mathrm{MDC}_{\mathrm{SCAN}}$ and hot spot idenfication considerations are then become the driver for sample spacing and respective DCGL $\mathrm{EMC}_{\text {. }}$

6. The ROCs being considered in the design include HTDs with higher analytical costs typically requiring wet chemistry and the site uses composite sampling to reduce analytical costs.

7. A licensee may choose to perform composite sampling during characterization or to provide additional FSS Class 2 and 3 survey unit coverage that ensures proper classification of the unit; however, comparison of the results must be to a modified investigation level (MIL) that is a fraction of the DCGL . The MIL must be based on DCGL $L_{W}$ in this case because Class 2 and Class 3 survey units should not have residual contaminant concentrations in excess of the $\mathrm{DCGL}_{\mathbb{W}}$ when properly classified. Under most FSS conditions, there is limited, if any benefit to composite sampling in properly classified Class 2 or 3 FSS survey units.

8. Composite sampling may be considered for HTDs for which an actual $\mathrm{MDC}_{\mathrm{SCAN}}$ cannot be established—e.g., pure beta or alpha emitter in soil—and there are no surrogate radionuclide relationships available. The composite sampling is used as a method to increase the probability of hot spot detection and as a means to reduce analytical cost. However, this situation would require considerable evaluations performed on a case-by-case basis. As such, this guidance provides only a general scenario and the associated variables.

9. Composite sampling may be used when maintaining sample density is important, yet one of 
the user's objectives is to cover a larger area without increasing the analytical budget.

Each of the above applications will require more rigorous data assessments, and may require re-testing of the individual increments comprising a composite in certain cases. These case requirements are discussed individually in Section 4.

\subsubsection{General Considerations}

The justification for incorporation of composites into a sampling plan relies upon several factors. Factors that will assist in properly using composites are discussed below.

1. Composite sample data may be applied without modification when information on individual samples is not important for the decisions that will be made with the data. When a threshold concentration or investigation level (IL) is of importance, for example, the DCGL $_{\mathbb{W}}$ during characterization or an FSS Class 2 survey unit or a DCGL $L_{\text {EMC }}$ in a Class 1 FSS unit, then a MIL must be established. The MIL will be a fraction of the concentration threshold based on the number of increments $(k)$ that comprise the composite. There are at least two options for setting the MIL limits. In most of the literature, the MIL is commonly defined as IL/k. Selection of the MIL value is critical. Too high of an MIL may result in missing discrete samples that exceed the IL (false negative). Too low of an MIL will conversely result in incorrectly investigating composite results that did not contain increments exceeding the IL (false positives). That is, a high false positive rate would be expected in cases where either $k$ is too high for the site conditions, there is not an expected substantial difference between the IL and the estimated site concentrations, or a combination of the two. The default MIL proposed under most conditions should be established:

$$
\mathrm{MIL}=I L / k
$$

Revisions to the default MIL determination would require technical justification. An example might be the method proposed by R. Correll (Correll 2001) where an MIL calculation of $I L / \sqrt{ } k$ is suggested as a means to reduce the number of false positive results relative to the $I L / k$ rule, yet minimize false negatives. The primary basis for proposing a revision is due to the conservativeness of the default MIL calculation. In either case, the site must provide 
both a lower and upper bound of the MIL for which definitive decisions may be made regarding a given result being definitely below or above a set action level.

2. Composites are useful when analytical costs are high, otherwise composite sampling is generally considered not to be cost effective. Furthermore, additional costs associated with forming composites and packaging and maintaining the increments is factored into the cost differential, prior to determining the value added of composite sampling.

3. Composite sampling is properly used when it will not impact the analyte integrity; it should not be used, for example, for volatile analytes.

4. The sample matrix must be amenable to homogenization and each increment must be equally represented in the composite.

5. Analytical detection limits and/or background interferences must be sufficiently low, relative to the proposed MIL, such that the probability of misclassifying a composite sample result and obtaining a result less than the MIL is negligible. An example for illustration would be if the Sr-90 NRC screening level DCGL $\mathrm{W}_{\mathrm{W}}$ of $1.1 \mathrm{pCi} / \mathrm{g}$ were the IL. A typical soil matrix analytical detection limit is $0.8 \mathrm{pCi} / \mathrm{g}$. Composite sample results would be expected to have a high false negative rate and should not be used unless the objective of the study is to only identify those areas of a site exhibiting high concentrations, as discussed in Section 4.5.

\subsubsection{Limitations}

Radiological survey plans that include composite sampling should be reviewed to ensure that the following inherent limitations are either addressed in the plan or the limitation will not adversely impact the data decisions.

1. Reduction in the information on variability. This limitation is potentially detrimental for situations such as determination of upper confidence levels; calculating a sufficient number of samples that will satisfy statistical power requirements and data quality objectives for hypothesis testing; and others.

2. Potential loss of temporal or spatial information. In some scenarios specific knowledge of the concentration in a unit area smaller than that represented by the composite is important 
in the decision process; for example, for differentiating classifications of a site.

3. Difficulty in homogenizing matrices. Such difficulty may be anticipated with clay soils and/or in cases where the contaminant is present as small particles that cannot be uniformly distributed.

4. Lost information on maximum concentrations. This limitation is important for threshold investigations and may be counteracted with appropriate protocols for re-testing of the questionable composite sample’s individual increments.

5. Lost information on concentration correlations for two or more ROCs. Therefore, composite sampling is not appropriate for determining surrogate relationships, such as estimating the mean surrogate correlations.

\subsection{Composite Sampling Plan and Data Quality Assessment Review Items}

The review of sampling plans proposing composite sampling would include ensuring that the licensee has addressed the following items.

1. The composite sampling design is implemented following proper procedures/project specific instructions. American Standards and Testing Materials (ASTM D 6051-96, Reapproved 2006) Standard Guide for Composite Sampling and Field Subsampling for Environmental $W$ aste Management Activities may be referenced for procedural considerations. Each composite increment is collected and a representative aliquot from each increment is containerized for possible reanalysis. The remaining portions of each increment are homogenized and the composite sample containerized. It is necessary that each increment contribute an equivalent volume/weight to the composite. In most cases there will be an original sample size $(N)$ that is based on statistical parameters, budgets, required sample coverage per unit area or other factors. The intent of the composite design is to lessen the costs and resources required to analyze and evaluate the data for $N$ samples by forming a lesser number of composite samples $(n)$. 
2. Consequences of the composite limitations described in Section 3.1.3. are reviewed.

3. The objective of the composite protocol is addressed and how the resultant data will be assessed.

4. The bases for determining $k$ per composite sample are determined. There are several factors that are considered. These include, but are not limited to, the physical nature of the samples, the anticipated concentrations of the ROCs relative to the detection limits (the dilution of a discrete action level results from the change from discrete samples to composites), and the capability to combine and homogenize them adequately. The larger $k$ becomes, the more difficult it will be to adequately homogenize the increments and therefore increases the introduced sampling error. In order to better control the sampling error, there should be a limit to the number of increments. Additionally, the analytical detection limit (d) will also impact the value of $k$ for a composite sample. This value is defined as:

$$
k<I L / d(\mathrm{EPA} 1995)
$$

Further guidance on the optimal value for $k$ is driven by two additional factors. These are 1) the ratio of the analytical cost to composite acquisition costs, and 2) the estimated ratio between the "error variability" component of the sample collection and measurement processes and the "inherent concentration variability" component. The guidance for optimal increment determination is detailed in the U. S. Environmental Protection Agency's Quality System Document QA/G-5S (EPA 2002) and the summarized considerations for $k$ that must be evaluated are several-fold. These factors for consideration and review include the following:

a. The analytical MDC (minimum detectable concentration) relative to the $\mathrm{DCGL}_{\mathbb{W}}$ and/or MIL.

b. The ratio of the measurement error standard deviation (includes random error in sample collection and compositing and the measurement error) to the inherent variation standard deviation (variability in the concentration of the target population). The optimal value for $k$ will decrease as this ratio approaches unity. With increasing values of $k$, it becomes increasingly difficult to prepare a 
representative composite. Therefore, this guidance recommends minimizing $k$ in most circumstances to between 3 and 6 , with a maximum of 10 .

c. The site should provide quality control results that demonstrate the error in composite formation is minimized. This may be demonstrated via a matrix spike to one of the increments forming a composite. The analytical result for the matrix spike composite sample should approximate $1 / k$ times the matrix spike concentration.

d. The ratio of the per sample analysis cost and the per sample collection and handling costs.

Once an optimal $k$ is selected, the number of composite samples $(n)$ analyzed will be:

$$
n=N / \text { optimized k. }
$$

5. The plan may also require specifying the retesting protocols. These retesting protocols are required whenever a threshold parameter is involved. In these cases, the plan must include a composite sample MIL at which point retesting may be required. In most cases, the MIL will be a fraction (as a function of $k$ as discussed in Section 3.1.2.) of what the IL would have been if composite sampling had not been considered; normally the IL would be the DCGL

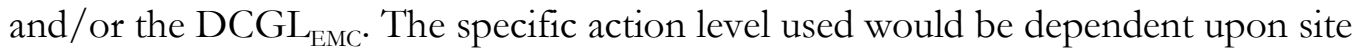
conditions, such as survey unit classification, sample spacing, $\mathrm{MDC}_{\mathrm{SCAN}}$, etc. A composite sample result greater than the MIL would require the licensee to investigate the result and possibly analyze the composite sample increments. Specifics of the MIL are provided within the examples.

6. Assurance must be provided that the MIL is greater than the analytical MDC and any background contributions.

7. When used during an FSS, the composite data are evaluated collectively to provide the estimate of the mean/median concentration levels in each survey unit, and individual composite results are compared to the respective $\mathrm{DCGL}_{\mathrm{EMC}}$ for the composite sample spacing, and are used in the statistical tests. This combined with the MIL provides the first level of assurance that allowable residual concentration criteria have been satisfied. 
8. Composite increments are maintained individually for re-testing.

9. When composite sampling is used to provide an increased probability of hot spot detection, the plan review will ensure that both the maximum hot spot size and concentration of concern have been carefully addressed and received regulatory approval prior to implementation of the plan. One item of note is that as the areal extent of contamination increases, the composite sampling economic benefits decrease. Further hot spot considerations are provided in Section 4.5.

10. The licensee's survey results reporting must provide a clear assessment of the individual and collective composite sample results to ensure compliance with allowable residual concentration commitments.

\section{COMPOSITE SAMPLING DESIGNS: SURVEY PLANNING EXAMPLE EVALUATIONS}

\subsection{MEAN CONCENTRATION ESTIMATION}

A licensee may choose to use composite sampling during site investigations to assist decision making in estimating the mean concentration. The reason(s) for doing so may be to reduce overall analytical costs and/or to increase site coverage. The target population of interest could range anywhere from the site as a whole to a specific survey area/unit, dependent upon the decommissioning phase. Implementation of composite sampling for mean estimation may be considered during any phase of the site decommissioning, including the FSS. Because both the MARSSIM-recommended Sign and Wilcoxon Rank Sum non-parametric statistical tests are an evaluation of the mean/median survey unit concentration relative to the DCGL $L_{\mathbb{W}}$, composite sample results may also be applied to the hypothesis test.

When an estimate of the study area mean is the sampling goal, the sample plan-ordinary random, systematic, stratified, etc.- - is selected based on the expected distribution of the ROChomogenous, heterogenous, spatially-related—and the overall DQOs. The number of samples $(n)$ necessary to estimate the mean objective are then calculated in accordance with the anticipated data use end-point. 
However, should the data be intended for other uses in addition to the mean estimation, the user is reminded of the loss of study area variability information with composite samples. Because the overall variability may be underestimated, a sufficient sample population may not be collected, increasing the risk of a decision error due to insufficient power. As such, consideration should be given to the total number of composite samples $(m)$ and the number of increments $(k)$ in each composite. A better estimate of the total degree of variability within the study area will be realized by adjusting the sample population from which the variability is calculated with a reduction in $k$ and/or an increase in $m$.

The approach of using composite samples to estimate the mean concentration within a study area should be reviewed to ensure that the licensee has provided the information or commitments listed below.

1. When the composite data are only intended to provide an estimation of the mean concentration, information on the individual increments is not important. The mean is calculated using the standard expression(s). However, if an IL for an individual increment is a project requirement, two conditions must be satisfied.

- A MIL must be determined.

- Individual increments must be maintained for re-analysis as necessary.

2. When the data are intended for both estimating the mean and determining if a threshold could be exceeded, the reviewer must also ensure that other conditions are satisfied. These begin with determining if the MDC of the selected analyses are several factors less than the expected ROC concentrations or IL, such that the concentrations of interest are not masked by limited analytical sensitivity. An example of such a condition and assumed parameters follows:

- $\quad$ ROC $=$ Th-230

- $\quad$ Discrete sample IL $=10 \mathrm{pCi} / \mathrm{g}$

- Selected analysis: gamma spectroscopy with a 4-hour count time

- Analytical MDC $=5 \mathrm{pCi} / \mathrm{g}$

- $\quad k$ per composite $=5$ 
- With the discrete sample IL of $10 \mathrm{pCi} / \mathrm{g}$, the analytical result must be capable of identifying composite samples that exceed the MIL.

- The default MIL is calculated as the $10 \mathrm{pCi} / \mathrm{g}$ action level divided by the number of composite increments, or $10 / 5=2 \mathrm{pCi} / \mathrm{g}$.

- Because the analytical MDC is greater than the IL and calculated MIL, composite sampling could not be applied without a more sensitive analysis, such as alpha spectroscopy.

- Alternatively, $k$ is reduced to 2 whereby the default MIL becomes $10 / 2=5 \mathrm{pCi} / \mathrm{g}$.

- These concepts may also be applicable for the other data end uses discussed in this guidance.

\subsection{IDENTIFICATION OF A ROC PRESENCE/AbSENCE Trait}

Early in the site decommissioning process, composite sampling may be beneficial to assist the user in determining the proportion of the site or other population sub-set that exhibits a particular trait. Two specific examples include areal ROC differentiation across the site and area classification. The resultant data may be used to provide an estimate of the proportion of the population that exhibits a specific trait and when additional testing is necessary.

1. This approach could be used to establish what proportion of the site could be considered for varying classifications. A simple example is determining the approximate proportion of a site that is impacted above the analytical detection limit for a ROC that is not present in background.

2. Identification of which ROCs are impacting site populations when the specific ROCs impacting a site are expected to vary over distinct site regions.

With the objective being a presence/absence trait, a binomial distribution may be applied and the number of positive composite results can be used to estimate the proportion of the site with the trait of interest. The estimated proportion $(p)$ of the population with the trait is a function of the positive results $(x)$ among $m$ composite random samples. $p$ is $\approx x / m$. The number of positive results is dependent upon k. This interrelationship may therefore be expressed as:

$$
p=1-(1-x / m)^{1 / k}(\text { EPA 2002) }
$$


This approach may also be further expanded to assist in the data gathering required to assess the presence/absence trait of hot spots of HTDs within a specified unit area. The following considerations must be agreed upon with the regulator.

1. Determine the a priori hot spot size and concentration of concern based on a dose consequence evaluation.

2. Specify the probability of not hitting a hot spot of the determined size; defined as $1-\beta$, the acceptable consumers risk is $\beta$.

3. Determine probability of the existence of hot spots based on results of sampling.

These are the sample planning considerations. Once the plan is developed, there will be further evaluations and considerations that shall include statistical inferences relative to probabilistic determination of the hot spot maximum size that could be missed by the plan design. Regulatory concurrence of unidentified hot spots of concern would therefore be necessary. Once data are evaluated, further determinations can be made as to the probability of any hot spots being in the survey area and statistical inferences determined as to the maximum probable level. A suggested approach for bounding this maximum probable concentration is to apply Chebyshev's inequality. Chebyshev's inequality guarantees that no more than $1 / \mathrm{k}^{2}$ of the distribution's values can depart more than $\mathrm{k}$ standard deviation from the mean (in this instance $\mathrm{k}=$ any real number $>0$ rather than, as previously defined, the number of increments). From the maximum level and size inference, the dose consequence may be calculated and further assessed.

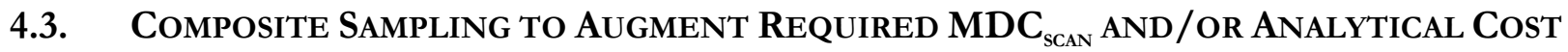 REDUCTION DURING Final Status Surveys}

A site may choose composite sampling as a means to resolve an FSS scenario where satisfying the required $\mathrm{MDC}_{\mathrm{SCAN}}$ for the applicable $\mathrm{DCGL}_{\mathrm{EMC}}$ results in an inordinate number of samples and an associated increase in sample analytical costs, which can be prohibitive. Other decommissioning survey phases may also select this approach to reduce the analytical costs.

Composite sampling can be used to satisfy the sample spacing requirements for the detection of hot spots of concern in Class 1 FSS survey units for cases where it proves difficult to achieve the 
required $\mathrm{MDC}_{\mathrm{SCAN}}$ conditions. The sample plan must provide the details which demonstrate that full consideration has been given to conditions that could deleteriously impact the decision making process.

An example of the approach is presented here.

- $\quad \mathrm{ROC}=\mathrm{Pu}-238$

- DCGL $_{\mathbb{W}}=31 \mathrm{pCi} / \mathrm{g}$

- Gamma $\mathrm{MDC}_{\mathrm{SCAN}}$ (FIDLER NaI detector $)=740 \mathrm{pCi} / \mathrm{g}$

- $\quad$ Survey unit area $=2000 \mathrm{~m}^{2}$

- The Sign test has been selected

\begin{tabular}{|c|c|c|c|c|}
\hline \multicolumn{5}{|c|}{ Table 4.1. Pu-238 Parameters } \\
\hline \multicolumn{5}{|c|}{ Pu-238 Table of Area Factors (AF) } \\
\hline $10 \mathrm{~m}^{2} \mathrm{AF}$ & $20 \mathrm{~m}^{2} \mathrm{AF}$ & $50 \mathrm{~m}^{2} \mathrm{AF}$ & $100 \mathrm{~m}^{2} \mathrm{AF}$ & $200 \mathrm{~m}^{2} \mathrm{AF}$ \\
\hline 104.8 & 54.0 & 22.1 & 11.2 & 5.6 \\
\hline \multicolumn{5}{|c|}{ Pu-238 DCGLEMC (pCi/g) } \\
\hline $10 \mathrm{~m}^{2}$ & $20 \mathrm{~m}^{2}$ & $50 \mathrm{~m}^{2}$ & $100 \mathrm{~m}^{2}$ & $200 \mathrm{~m}^{2}$ \\
\hline 3,249 & 1,674 & 685 & 347 & 174 \\
\hline
\end{tabular}

For this FSS example, assume a site's DQO planning inputs result in 15 required samples. The respective area represented by each sample is therefore $2000 / 15$ or $133 \mathrm{~m}^{2}$. The interpolated area factor from Table 4.1 for $133 \mathrm{~m}^{2}$ is 8.4 . The 8.4 area factor corresponds to a DCGL $\mathrm{EMC}_{\text {of }}$ $260 \mathrm{pCi} / \mathrm{g}$. The actual $\mathrm{MDC}_{\mathrm{SCAN}}(740 \mathrm{pCi} / \mathrm{g})$ is compared with this required scan MDC $(260 \mathrm{pCi} / \mathrm{g})$. Because the actual $\mathrm{MDC}_{\mathrm{SCAN}}$ exceeds the required $\mathrm{MDC}_{\mathrm{SCAN}}$ activity concentration level, sample spacing must be reduced to account for potential hot spots. The area factor necessary to satisfy hot spot considerations is calculated: $\mathrm{MDC}_{\mathrm{SCAN}}\left(\right.$ actual) $/ \mathrm{DCGL}_{\mathrm{W}}=23.9$. This area factor equates to a sample spacing of $45.7 \mathrm{~m}^{2}$. Therefore, 44 samples would be necessary to ensure the $\mathrm{MDC}_{\mathrm{SCAN}}$ is adequate to detect hot spots of the corresponding magnitude.

The site requests that sample sizes remain essentially equivalent to the original plan, due to the costs associated with analyzing 29 additional samples in multiple Class 1 survey units. Therefore, 
composite sampling and retesting are proposed and factored into the FSS plan in order to minimize the additional analytical costs. To do so, the DQOs must be planned accordingly and included in the decision making process. This example is further developed below as an illustration.

The initial 15 sample design is shown in Figure 4.1a. However, hot spot considerations require the sample size be increased to 44 . The original 15 sample design is maintained by forming composite samples composed of 2 to 3 increments each as represented in Figure 4.1b. The revised design sample spacing $45.5 \mathrm{~m}^{2}$, the three increment composite therefore represents a sample area of approximately $136.4 \mathrm{~m}^{2}$. This design will provide the necessary assurance that any hot spots of concern will be identified either by sampling or by scanning. Each of the 44 increments are collected, composites formed, individual increments are maintained for retesting as needed, and the composites are analyzed. The composite results are then compared with the appropriate MIL. A composite result less than the MIL provides the evidence that hot spots are below the action threshold and composite results greater than the MIL will require retesting of the increments before a final determination is made as to whether further investigations are required. 


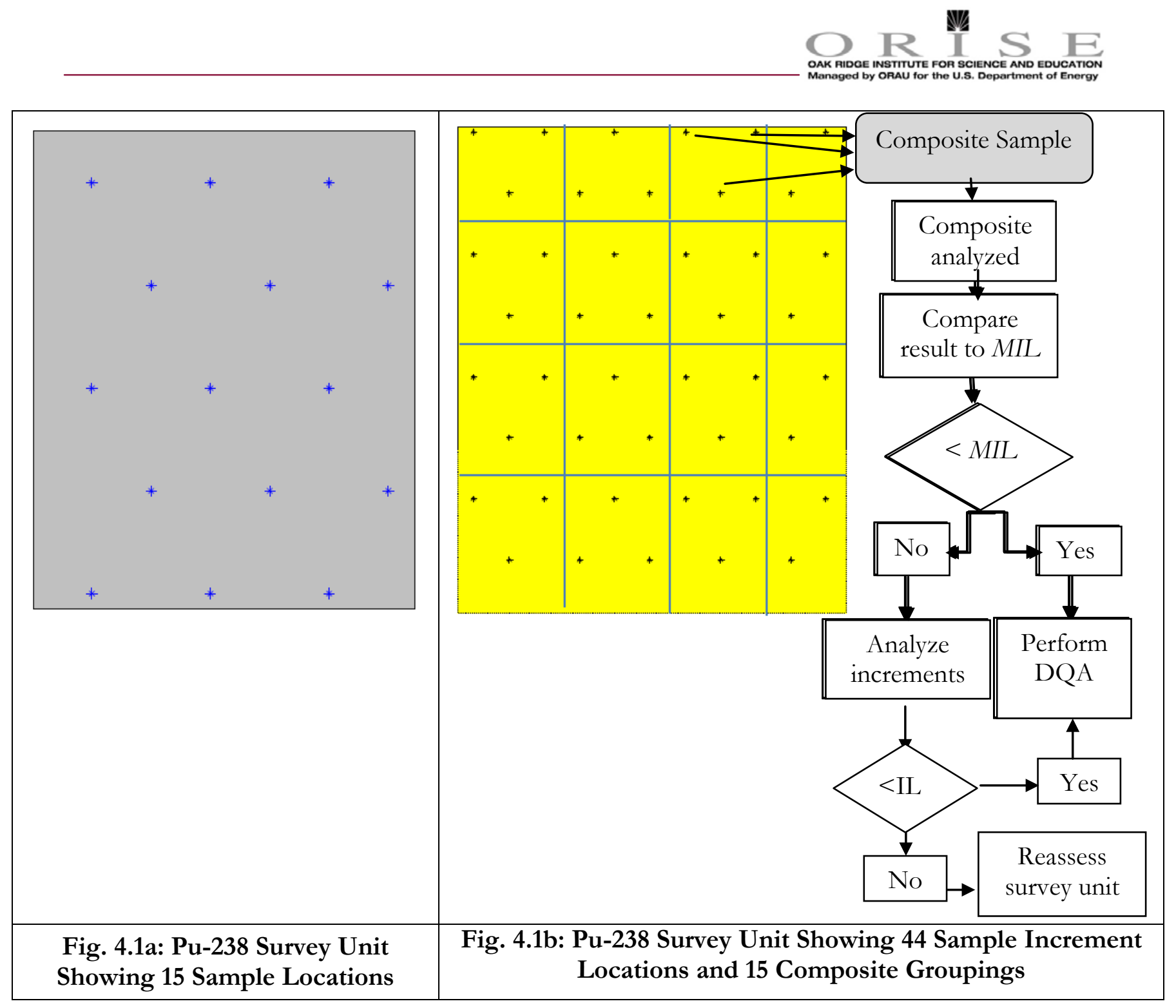

Fig. 4.1. Pu-238 Sample Number Comparison

The data quality assessment phase will require added rigor to close the differential between the required $\mathrm{MDC}_{\mathrm{SCAN}}$ that was addressed by increasing the sampled locations to 44 and forming the 15 composites. The data assessment would compare each composite concentration result with an appropriate MIL that accounts for the various scenarios that could exist. There are multiple combinations of increments adding elevated concentrations of the ROC to the composite results. For this example the scenarios to consider for an MIL exceedance could be:

1. 1 increment adding a high concentration

2. 2 increments adding moderate to high concentrations

3. All 3 increments adding low to high concentrations 
These assessments will lead to varying decisions, including no further investigation necessary, failure of the Elevated Measurement Comparison, or retesting of increments and comparison of the results

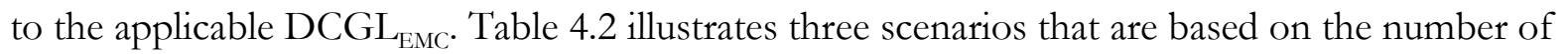
contaminated (involved) increments for a composite sample. The scenarios show the MILs that are a function of the area represented by the number of involved composite increments with $\mathrm{Pu}-238$ concentrations that equal the respective $\mathrm{DCGL}_{\mathrm{EMC}}$. The results show three differing concentration values where the potential exists for exceeding a $\mathrm{DCGL}_{\mathrm{EMC}}$. Because of these various scenarios, the composite sample MIL must be established at the lowest concentration value-or $244 \mathrm{pCi} / \mathrm{g}$ in the Table 4.2 example. Any composite result greater than $244 \mathrm{pCi} / \mathrm{g}$ would require retesting of the individual increments that comprised the suspect composite sample. The example assumes that any hot spots smaller than $45.5 \mathrm{~m}^{2}$ with activity levels greater than $740 \mathrm{pCi} / \mathrm{g}$ would be identified during the scanning phase of the survey.

\begin{tabular}{|c|c|c|c|c|c|}
\hline \multirow{2}{*}{$\begin{array}{l}\text { Area } \\
\left(\mathrm{m}^{2}\right)\end{array}$} & \multirow{2}{*}{$\begin{array}{c}\text { Area } \\
\text { Factors }\end{array}$} & \multirow{2}{*}{$\begin{array}{c}\text { DCGLEMC } \\
(\mathrm{pCi} / \mathrm{g})\end{array}$} & \multicolumn{2}{|c|}{$\begin{array}{c}\text { Involved Increments/ } \\
\% \text { Activity Weighted Contribution at the } \\
\text { DCGL }\end{array}$} & \multirow[t]{2}{*}{ MIL (pCi/g) } \\
\hline & & & $\begin{array}{l}\text { Number of } \\
\text { Increments }\end{array}$ & $\begin{array}{c}\% \text { Activity } \\
\text { Contribution }{ }^{1}\end{array}$ & \\
\hline 136.5 & 8.2 & 254 & 3 of 3 & 1 & 254 \\
\hline 91 & 12.2 & 378 & 2 of 3 & 0.67 & 253 \\
\hline 45.5 & 24 & 740 & 1 of 3 & 0.33 & 244 \\
\hline
\end{tabular}

$1 \%$ assumes other increment(s) have no added activity

The conclusion of the example is that for the established parameters, using the composite sampling approach will provide a high degree of certainty that both the average residual $\mathrm{Pu}-238$ can be readily determined and hot spots of concern will be identified with minimal false negatives when composite sampling with a defensible MIL is combined with surface scanning. Lastly, the analytical cost for the survey was maintained, although there will be additional field labor costs to collect, package, and record the composite sample and the increments. 


\subsection{The Contaminant DCGL ${ }_{\mathrm{w}}$, Mean, VARiability, AND/OR Decision Error Combinations Result in a RELATIVE SHIFT $<1$}

MARSSIM recommends that the relative shift be maintained between a value of 1 and 3 such that a reasonable sample size results during the FSS. Site-specific conditions could lead to situations where the relative shift is less than one, requiring a large number of samples $(N)$ to achieve adequate statistical power. Such conditions, either individually or in combinations, could include a high expected mean concentration (lower bound of the gray region [LBGR]) relative to the $\mathrm{DCGL}_{\mathrm{W}}$ and/or a high degree of variability within the survey unit. Current MARSSIM guidance recommends the following when this situation occurs: either reducing the value of the LBGR, which impacts the concentrations at which a Type II error could occur, or re-visiting the DQOs together with the regulatory authority.

The basic two options available to the site for revising the DQOs such that sample sizes are reduced—other than reducing the LBGR — are increases in either the DCGL $\mathrm{W}_{\mathbb{W}}$ or the Type I error. Both options require regulatory interactions and approvals. Another option that a site may consider is composite sampling, where the initial MARSSIM designed sample size forms the basis for the number of increments $(k)$. The site would therefore be required to determine the number of composite samples that will be a function of an optimal $k$ value. The considerations for $k$ that must be evaluated have been provided in Section 3.2.

\subsection{Classifying Survey Areas as Containing Hot Spots}

Increased probability for hot spot detection was introduced in Section 4.2, although when not performed correctly, the opposite effect could occur where a hot spot is masked. The advantage for hot spot detection using composite samples is that through the reduced analytical costs that can be achieved, more of the budget is available for sampling. This directly leads to better areal coverage and hence increases the probability that a sample location will fall on a hot spot. Therefore, a composite sample approach can provide greater confidence for detecting hot spots of those ROCs considered to be HTDs such as Sr/Y-90, C-14, etc. when a surrogate is not available. Therefore, an affordable FSS plan can only be developed by first deciding upon an acceptable hot spot size and concentration magnitude. 
Again, the emphasis in this guidance is that the integrated MARSSIM design for detecting HTD hotspots is not sufficient and requires that the site present, for regulatory approval, a proposed approach that combines the required number of samples for the statistical tests and for addressing hot spots. For this guidance, such a technical approach might include either composite sampling, composite sampling further supplemented with Rank Set Sampling, or the adaptation of a probability-based design for locating hot spots of a predetermined size and shape (EPA 2002, Patil 2002b, Jozani and Johnson 2010, Gilbert 1987). However, before preparing either design, an a priori hot spot size of concern and the associated DCGL $\mathrm{EMC}_{\mathrm{C}}$ must be determined. Once the hot spot size is determined, then either a discrete or composite sampling approach can be applied to provide a high level of probability that the hot spot will be sampled.

There remains yet another problem associated with this a priori hot spot. The problem is how to select the a priori size as there will likely remain stakeholder concerns for smaller hot spots that could again be missed. Therefore, the technical justification must also include additional dose modeling details as to the impacts from any other small hot spots that could go undetected and potential contribution to the total dose from all remaining source terms across the site. Other approaches that may be considered include maximum concentration bounding scenarios using Chebyshev's inequality or a Bayesian approach to estimate the maximum potential contamination level at the site.

In summary, the HTD hot spot identification condition will require significant evaluation and discussions with appropriate survey design and regulatory authorities.

\section{CONCLUSION}

In a "typical" final status radiological survey, the reduced probability of identifying hot spots is accounted for through the iteration of adjusting sample spacing to satisfy required scan MDC sensitivity. To limit the increases in sample sizes, more sites are suggesting composite sampling as one method to control the increased analytical costs that result. As with most sampling approaches there are both advantages and disadvantages that require evaluation and review to ensure that any limitations are accounted for in the plan.

With this information, robust radiological sampling plans can be developed that will address multiple issues while providing assurance regarding decisions as to the average residual concentrations across 
the survey unit and hot spot considerations as well. The number of composite samples and composite increments can be controlled based on type of contaminant, DCGL $\mathrm{W}_{\mathrm{W}}$ levels, analytical MDCs, and the corresponding appropriate MILs. Additional guidance on optimizing all factors may be found in the references. 


\section{REFERENCES}

ASTM International 2006. Standard Guide for Composite Sampling and Field Subsampling for Environmental Waste Management Activities. Designation: D 6051-96. West Conshohocken, PA. October 1.

Carson, Jr., J. H. 2001. Analysis of Composite Sampling Data Using the Principle of Maximum Entropy. Environmental and Ecological Statistics, Volume 8, pp 201-211. Kluwer Academic Publishers.

Correll, R. L. 2001. The Use of Composite Sampling in Contaminated Sites-A Case Study. Environmental and Ecological Statistics, Volume 8, pp 185-200. Kluwer Academic Publishers.

EPA 1995. EPA Observational Economy Series; Volume 1: Composite Sampling, EPA-230-R-95-005. U.S. Environmental Protection Agency. Washington, DC. August.

EPA 2002. Guidance on Choosing a Sampling Design for Environmental Data Collection for Use in Developing a Quality Assurance Project Plan, EPA QA/G-5S. U.S. Environmental Protection Agency.

Washington, DC. December.

Gilbert, R. O. 1987. Statistical Methods for Environmental Pollution Monitoring. Van Nostrand Reinhold.

J.E.Jozani, M. J. and B. C. Johnson 2010. Design Based Estimation For Ranked Set Sampling in Finite Populations. Environmental and Ecological Statistics. Springer Science+Business Media, LLC. Published on-line September 5.

NRC 1998. A Nomparametric Statistical Methodology for the Design and Analysis of Final Status

Decommissioning Surveys, NUREG-1505; Revision 1. U.S. Nuclear Regulatory Commission. Washington, DC. June.

NRC 2000. Multi-Agency Radiation Survey and Site Investigation Manual (MARSSIM), NUREG-1575;

Revision 1. U.S. Nuclear Regulatory Commission. Washington, DC. August.

NRC 2006. Consolidated Decommissioning Guidance, NUREG-1757; Revision 2. U.S. Nuclear Regulatory Commission. Washington, DC. September.

Patil, G. P. 2002a. Composite Sampling. Encyclopedia of Environmetrics, Volume 1, pp 387-391. John Wiley \& Sons, Ltd, Chichester.

Patil, G. P. 2002b. Ranked Set Sampling. Encyclopedia of Environmetrics, Volume 3, pp 1684-1690. John Wiley \& Sons, Ltd, Chichester. 